\title{
Posterior Instability of the Glenohumeral Joint
}

\section{A Technique of Repair*}

\author{
Richard J. Hawkins, † MD, FRCS(C), and David H. Janda, $\ddagger \S M D$
}

\author{
From the †Steadman-Hawkins Clinic, Vail, Colorado, and the $¥$ Institute for Preventative \\ Sports Medicine, Ann Arbor, Michigan
}

\begin{abstract}
Seventeen patients underwent posterior capsulotendinous tensioning procedures to eliminate recurrent posterior glenohumeral instability. Fourteen patients were evaluated an average of 44 months (range, 18 to 98) after surgery. The average patient age was 27 years. Before surgery, all patients were unable to perform their activities of daily living, occupational activities, and athletic activities. Preoperatively, the average pain rating score on a visual analog scale was 5 of 10 at rest and 9 of 10 with activities. Six patients had previous anterior reconstructions. After surgery, the average range of motion was $174^{\circ}$ of forward elevation and $69^{\circ}$ of external rotation; internal rotation was to the thumb level of T-8. No patient had a recurrence of posterior instability. After surgery, the average pain rating score was 2 of 10 at rest and 4 of 10 with activities. All patients improved after their operations, but four patients were minimally disabled from activities of daily living; six patients experienced shoulder fatigue at work; and four patients had difficulty with sports activities. Overall, 13 of the 14 patients were satisfied with their surgical procedures and their outcomes.
\end{abstract}

Posterior instability of the glenohumeral joint continues to be one of the most intriguing and confusing pathologic conditions confronting orthopaedic surgeons and sports medicine specialists. The confusion with posterior instability of the glenohumeral joint begins with classifying the instability. Difficulties also include interpreting physical

*Presented at the 20th annual meeting of the AOSSM, Palm Desert, California, June 1994.

\$Address correspondence and reprint requests to David $\mathrm{H}$. Janda, MD Institute for Preventative Sports Medicine, POB 7032, Ann Arbor, MI 48107.

No author or related institution has received any financial benefit from research in this study. examination findings, designing nonoperative treatment, and, finally, deciding what operative intervention should be used when nonoperative treatment fails.

Classification of posterior instability of the glenohumeral joint can be subcategorized as follows:

1. Acute posterior dislocation, with and without impression defect.

2. Chronic posterior dislocation, locked (missed) with impression defect.

3. Voluntary recurrent posterior subluxation, habitual (willful, personality disorder) and muscular control (not willful).

4. Involuntary recurrent posterior subluxation, positional (demonstrable by the patient) and nonpositional (not demonstrable by the patient).

This manuscript addresses the more prevalent problem of recurrent posterior subluxation and, in particular, the voluntary nonhabitual form, as well as involuntary positional and nonpositional forms of posterior instability. ${ }^{2}$

Significant structural differences, which have been identified between the anterior and posterior aspects of the glenohumeral joint, must be recognized to appreciate the differences between anterior and posterior shoulder instability. One difference includes the posterior labrum, which does not provide the same support as the anterior labrum. The posterior labrum is loosely attached to the surrounding capsule posteriorly, with no direct posterior ligament reinforcement. O'Brien et al. ${ }^{4}$ and Warren et al. ${ }^{8}$ have shown that posterior stability is provided by anterior restraints such as the inferior, the middle, and the superior glenohumeral ligaments, and the rotator cuff interval. Harryman et al. ${ }^{1}$ have also shown that the superior coracohumeral ligament and interval are important contributors to posterior stability. Electromyographic activity of selected shoulder girdle muscles has demonstrated that the humeral head can be pushed backward with the deltoid and biceps muscles or pulled posteriorly by the infraspinatus muscle, the posterior deltoid muscle, or both. ${ }^{5}$ Inhibition of the scapular rotators, as determined with 
electromyography, accompanies those movements and this inhibition leads to winging of the scapula, which has been associated with posterior instability. ${ }^{5}$ Once recurrent posterior instability occurs, redundancy can develop in the posterior capsule (i.e., stretching of the capsule) and contributes to the ongoing instability. ${ }^{3}$

When nonoperative treatment fails, surgical treatment can be considered. In our experience, however, the majority of patients will respond to a nonoperative program. Operative intervention is reserved for the patients who do not habitually and willfully dislocate their shoulders as outlined by Rowe et al. ${ }^{7}$

Various surgical options have been employed to control posterior instability of the glenohumeral joint. Entities proposed include posterior bone blocks, subscapularis muscle and biceps tendon transfer, glenoid and humeral osteotomies, posterior Bankart repairs, posterior capsular plication and infraspinatus advancement, staple capsulorrhaphy, and the posteroinferior capsular shift. ${ }^{2}$ Having experienced failure with many of these procedures, we have developed and will present here a posterior capsulotendinous tensioning procedure to alleviate posterior instability of the glenohumeral joint.

\section{MATERIALS AND METHODS}

From June 1986 to July 1990, 17 patients with recurrent posterior glenohumeral instability underwent posterior capsulotendinous tensioning procedures to eliminate the pattern of instability. Fourteen patients were available at followup. The mean patient age was 27 years at surgery and the average followup was 44 months (range, 18 to 48 ). Nine surgeries involved left shoulders; five surgeries involved right shoulders. The cause of the instability was not related to a traumatic event in seven shoulders and was related to a traumatic event in the remaining seven shoulders including one from water skiing, two from diving, one from a fall, one from football, and two from motor vehicle accidents. The senior author $(\mathrm{RJH})$ performed the surgical procedures. Followup consisted of a patient interview and a physical examination. In addition to documenting range of motion and instability at followup, a questionnaire was distributed to reference the level of pain and the level of disability in activities of daily living, work, and sports activities.

All patients had pain and instability preoperatively. They were disabled in activities of daily living, often precluding laboring occupational and athletic activities.

\section{Operative Technique}

The patient was placed in a lateral decubitus position supported by a bean bag, prepared for surgery, and was draped with the injured arm free. We used a longitudinal incision that began $2 \mathrm{~cm}$ medial to the posterolateral corner of the acromion and coursed distally to the posterior axilla (Fig. 1A). The underlying deltoid muscle was then split in line with its fibers in a blunt fashion to reveal the underlying infraspinatus and teres minor muscles (Fig. 1B). A deltoid muscle retractor was placed over the humeral head and under the acromion. The arm was then placed in neutral rotation (Fig. 1C) and a vertical incision was made through the infraspinatus tendon and the capsule (Figs. 1D and 1E). The incision was parallel to and at the level of the joint line with the arm in neutral rotation. To avoid an injury to the axillary nerve the teres minor muscle was not violated (Fig. 1F). With the capsulotomy complete, stay sutures were positioned and the joint was visualized. A humeral head retractor may be placed to subluxate the head for better visualization. The joint was inspected, the retractor removed, and the arm was externally rotated, allowing the lateral stump of the capsule to be sutured into the intact posterior labrum (Fig. 1G). If the labrum had been detached, as occurred in one of our patients, the stump of the capsule and the tendon can be secured to drill holes or secured from anchors in the glenoid. The medial portion of the capsule and infraspinatus muscle were then sutured laterally over the previous repair. At this point, the arm should be able to rotate approximately $20^{\circ}$ internally. If the repair was made too tightly, the repair can be relaxed (i.e., the tissue in the repair is sutured to the posterior capsule, not the labrum).

No sutures were placed in the deltoid fascia and routine closure of the wound was performed. Before the patient awoke, the arm was placed in approximately $20^{\circ}$ of abduction and $20^{\circ}$ of external rotation, with the upper arm slightly posterior to the coronal plane. An orthosis or shoulder spica cast was then placed.

\section{Postoperative Management}

The shoulder of the patient was immobilized for 6 weeks if the injury was atraumatic in origin and for 4 weeks if the injury was traumatic. After 4 or 6 weeks, the orthosis was removed from the patient and a three-phase rehabilitation program was started. The first phase focused on passive range of motion exercises for 2 weeks. The second phase of the program, which consisted of active range of motion and a terminal stretching program for 4 weeks, was then implemented. The third phase consisted of a rotational and scapular strengthening program with ongoing terminal stretching. Realistically, 6 months of aggressive postoperative management may be needed before a satisfactory return to sports activities or heavy occupational tasks.

\section{RESULTS}

The range of motion postoperatively averaged $174^{\circ}$ of forward elevation and $69^{\circ}$ of external rotation with the arm at the side; the internal rotation was to the thumb level of T-8. Six patients who underwent a posterior reconstruction had undergone a previous anterior procedure. At followup, no patient had symptomatic recurrent posterior instability.

After surgery, four patients had minimal disability due to fatigue, but they were improved from their preinjury status. Six patients experienced shoulder fatigue while at work; yet, they returned to their regular jobs. Four patients had difficulty with sports activities because of 
A

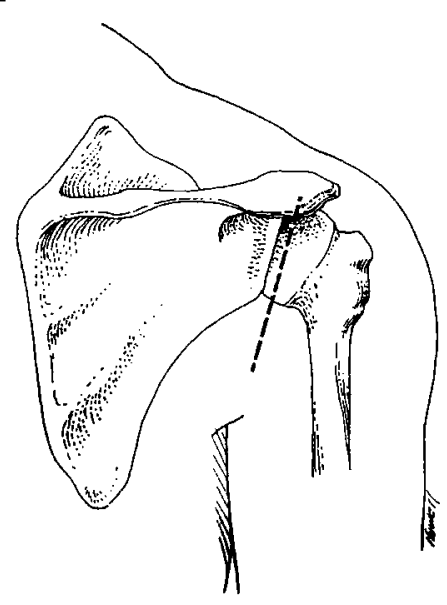

D
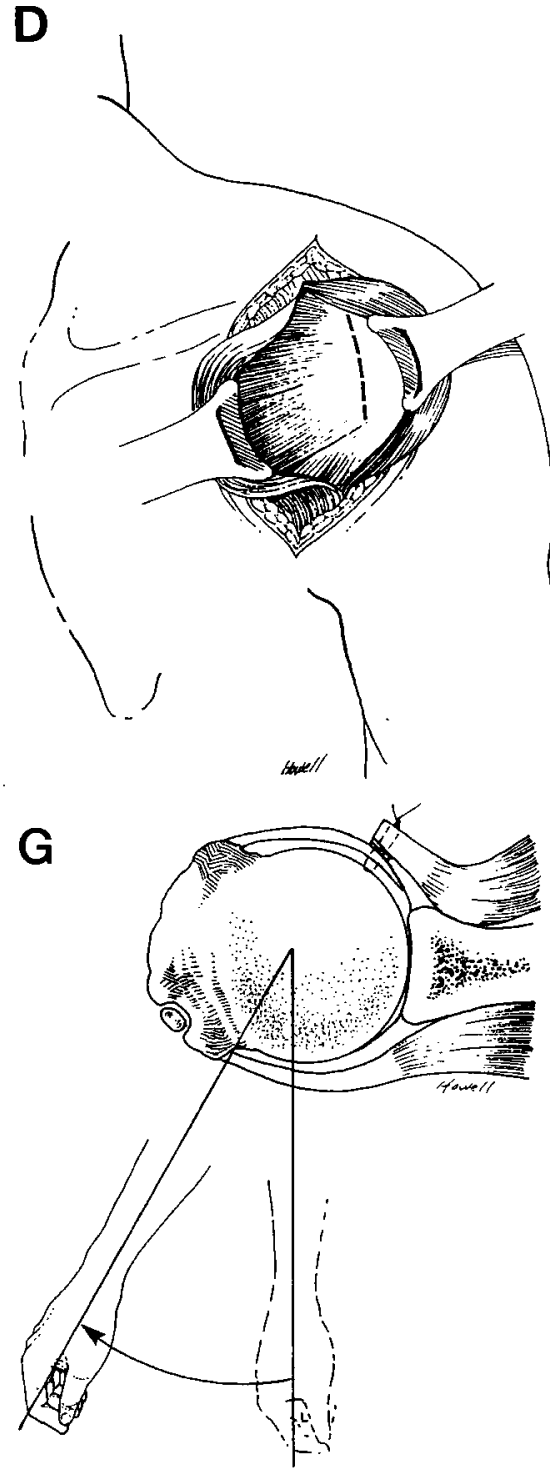

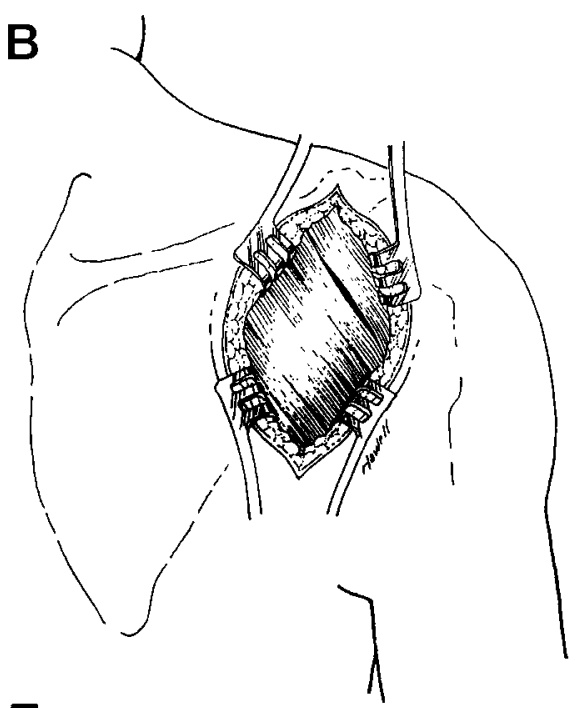

E

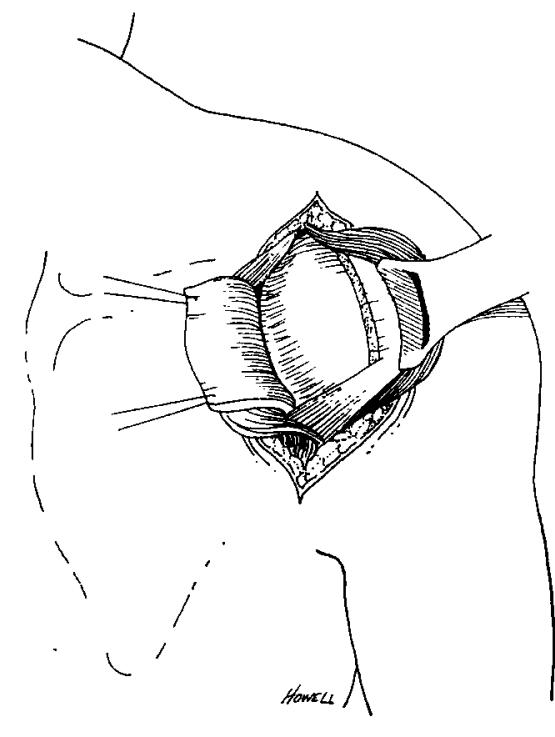

Figure 1. The posterior capsular tensioning procedure for posterior glenohumeral instability. See text for details.
$\mathbf{F}$

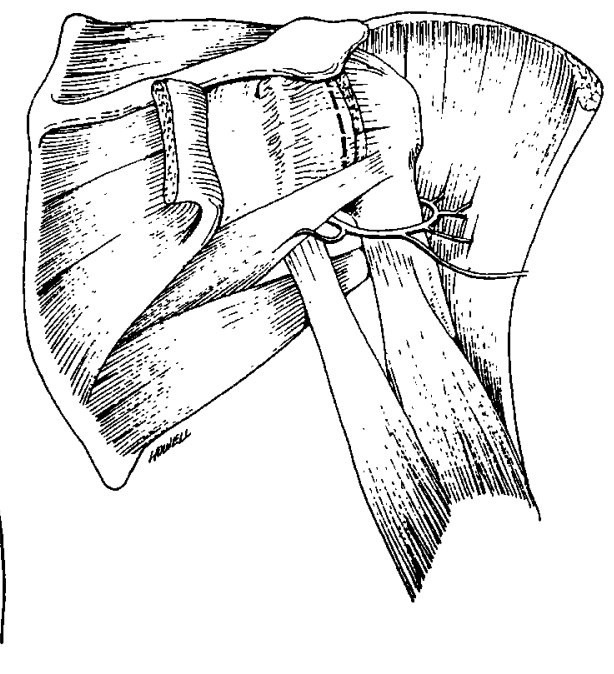

C

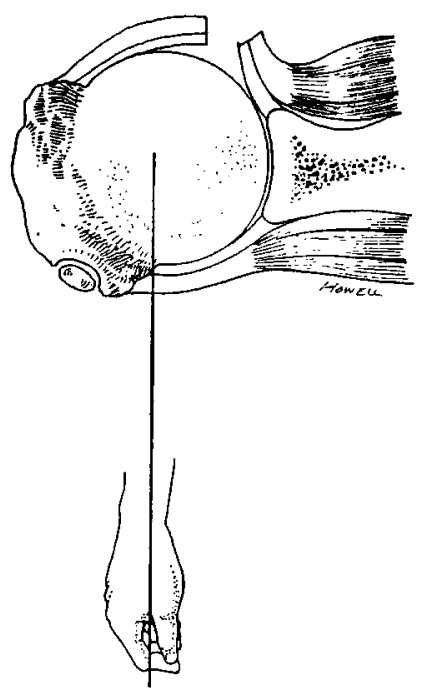


weakness and fatigue. Ten patients returned to their recreational sports activities.

The average pain rating scores on a visual analog scale were 5 of 10 at rest preoperatively and 2 of 10 at rest postoperatively. Preoperatively, the average pain rating score on a visual analog scale was 9 of 10 with activities, and postoperatively, the score fell to 4 of 10 with activities. After surgery, 13 of 14 patients were satisfied with their procedures and outcomes. The one patient who had a poor outcome continued to have pain and apprehension and did not return to recreational sports or strenuous physical activity.

\section{Complications}

After surgery, there were no neurologic or vascular injuries and there were no infections.

\section{DISCUSSION}

Historically, failure after repair for posterior instability has been fairly high. Hawkins et al. ${ }^{2}$ had a postoperative recurrence rate of approximately $50 \%$ (13 of 26 shoulders). A recent report by Pollock and Bigliani ${ }^{6}$ indicated an increased success rate with surgical reconstruction.

A successful outcome involves patient selection and, perhaps, improved techniques. This paper offers an alternative technique that we find comparatively effective in restoring posterior stability. Patients who have posterior subluxation of the glenohumeral joint that is unresponsive to nonoperative treatment may be considered for operative intervention. The majority of patients, of course, are treated with nonoperative physical therapy and do reasonably well.

We report on 14 patients who had an appropriate follow-up time (44 months postoperatively). After nonopera- tive treatments failed, these patients underwent procedures for posterior capsulotendinous tensioning to eliminate recurrent posterior glenohumeral instability. Overall, 13 of the 14 patients were satisfied with their surgical procedures. All of these patients indicated they would have the operations again.

\section{CONCLUSIONS}

The procedure described here is an imperfect operation in that not all patients returned to aggressive labor and work and several patients were unable to return to recreational sports activities. By and large, our procedure has proved to be the most successful operation we have performed. However, some patients continued to have a mild degree of discomfort in their shoulders, particularly with strenuous activities. The procedure for posterior capsulotendinous tensioning, as we described it, is a surgical option to consider if nonoperative treatment fails.

\section{REFERENCES}

1. Harryman DT II, Sidles JA, Clark JM, et al: The role of the coracohumeral ligament in passive motion and stability of the shoulder. Orthop Trans 14: 595-596, 1990

2. Hawkins RJ, Koppert G, Johnston G: Recurrent posterior instability (subluxation) of the shoulder. J Bone Joint Surg 66A: 169-174, 1984

3. Hawkins RJ, McCormack RG: Posterior shoulder instability. Orthopedics 11: 101-107, 1988

4. O'Brien SJ, Neves MC, Arnoczky SP, et al: The anatomy and histology of the inferior glenohumeral ligament complex of the shoulder. Am J Sports Med 18: $449-456,1990$

5. Pande $P$, Hawkins $R$, Peat $M$ : Electromyography in voluntary posterior instability of the shoulder. Am J Sports Med 17: 644-648, 1989

6. Pollock RG, Bigliani LU: Recurrent posterior shoulder instability. Diagnosis and treatment. Clin Orthop 291: 85-96, 1993

7. Rowe CR, Pierce DS, Clark JG, et al: Voluntary dislocation of the shoulder. A preliminary report on a clinical, electromyographic, and psychiatric study of twenty-six patients. J Bone Joint Surg 55A: 445-460, 1973

8. Warren RF, Kornblatt IB, Marchand DR: Static factors affecting posterior shoulder stability. Orthop Trans 8: 89, 1984 